\title{
A multisymplectic approach to defects in integrable classical field theory
}

\author{
V. Caudrelier ${ }^{a}$ and A. Kundu ${ }^{b}$ \\ ${ }^{a}$ Department of Mathematics, City University London, \\ Northampton Square, EC1V OHB London, U.K. \\ ${ }^{b}$ Theory Division, Saha Institute of Nuclear Physics, \\ 1/AF Bidhannagar, Kolkata 700064, India \\ E-mail: v.caudrelier@city.ac.uk, anjan.kundu@saha.ac.in
}

ABSTRACT: We introduce the concept of multisymplectic formalism, familiar in covariant field theory, for the study of integrable defects in $1+1$ classical field theory. The main idea is the coexistence of two Poisson brackets, one for each spacetime coordinate. The Poisson bracket corresponding to the time coordinate is the usual one describing the time evolution of the system. Taking the nonlinear Schrödinger (NLS) equation as an example, we introduce the new bracket associated to the space coordinate. We show that, in the absence of any defect, the two brackets yield completely equivalent Hamiltonian descriptions of the model. However, in the presence of a defect described by a frozen Bäcklund transformation, the advantage of using the new bracket becomes evident. It allows us to reinterpret the defect conditions as canonical transformations. As a consequence, we are also able to implement the method of the classical $r$ matrix and to prove Liouville integrability of the system with such a defect. The use of the new Poisson bracket completely bypasses all the known problems associated with the presence of a defect in the discussion of Liouville integrability. A by-product of the approach is the reinterpretation of the defect Lagrangian used in the Lagrangian description of integrable defects as the generating function of the canonical transformation representing the defect conditions.

KEYwords: Integrable Field Theories, Integrable Hierarchies

ARXIV EPRINT: 1411.0418 


\section{Contents}

1 Introduction 1

2 Multisymplectic structure of the NLS equation 3

2.1 Space and time Hamiltonian forms for NLS 3

2.2 Classical $r$-matrix approach for the two Poisson brackets 6

2.2.1 The standard approach with $\{,\}_{S} \quad 6$

2.2.2 Classical $r$-matrix approach for the new bracket $\{,\}_{T} \quad 8$

$\begin{array}{lll}2.3 & \text { Canonical transformations and Bäcklund transformations } & 10\end{array}$

3 NLS with a defect: Liouville integrability 12

$\begin{array}{lll}3.1 \text { Defects as frozen Bäcklund transformations } & 12\end{array}$

3.2 Liouville integrability: defect conditions as canonical transformations $\quad 13$

3.3 Liouville integrability: classical $r$-matrix approach with defect $\quad 14$

4 Concluding remarks $\quad 15$

\section{Introduction}

Real materials and systems as a rule exhibit defect structures. However, since the presence of defects usually spoils the regular behaviour predicted by the study of the ideal materials, in theoretical investigations one usually tries to avoid the problem of defects, which in general is difficult to handle. Nevertheless, since the presence of defects, even a single one, might play a pivotal role in determining bulk properties of a system, the investigation of defect problems has been undertaken quite intensively in recent years, both from theoretical and experimental point of view [1]-[2].

With the advent of experimental realisations of systems, the question of defects, that has been thought to be of purely academic interest for decades [3]-[6], emerged naturally in realistic models in cold atoms and optical setups, which could be described efficiently by $1+$ 1 dimensional integrable systems. The initial focus was mainly in quantum field theories [7, $8]-[12,13]$ and was concentrated until quite recently [14]-[16] on various quantum systems. However, it has been understood soon, that in certain cases the presence of defects may be considered in an exact manner, preserving even the integrability of the models. The general framework that includes most of the previous studies was proposed comprehensively in [17].

The question of integrable defects in classical field theories was considered almost ten years after the publication of the first paper on the topic in integrable QFT. In a series of papers $[18,19]-[20-24]$ related to several key models like the sine-Gordon model, the NLS equation, etc, a Lagrangian approach was proposed where a contribution from the defect is required to compensate for the loss of conservation of the momentum due to the presence of 
a defect. It was argued that this is enough to ensure the integrability of a defect model. A crucial observation to support this was that the conditions on the fields that one obtains in this way correspond to Bäcklund transformations frozen at the location of the defect. This approach triggered a strong activity in the analysis of the defect in integrable classical field theories. The observation on frozen Bäcklund transformations was fully exploited in [25] in conjunction with the Lax pair formulation of the general AKNS approach [26] to obtain a generating function of the entire set of modified conserved quantities. This also allowed to answer some questions left open in the Lagrangian formulation like the formulation of the defect conditions directly in terms of the fields of the theory for models like KdV. It also settled the question of integrability in the sense of the presence of an infinite number of conserved quantities. But soon, the question of Liouville integrability became a main issue. The sine-Gordon model was the first model to receive attention [27], followed by a very nice series of papers tackling the question systematically for several models [2831]. The procedure in these investigations is based on the a priori assumption that the defect matrix satisfies appropriate Poisson bracket relations formulated in the context of the classical $r$-matrix approach. A careful regularization is needed in this procedure which yields the so-called "sewing conditions" between the fields in the bulk and those contained in the defect matrix. The consistency of the approach must then be checked a posteriori.

However, there still exist two points of view, that have not been reconciled so far. On the one hand, the defect matrix (or operator) may be given as a Bäcklund matrix involving the values of the fields at the defect point [25] but the Hamiltonian picture in this setting has not been understood so far. On the other hand, one may start a priori with a Hamiltonian structure given by an $r$-matrix and require that the defect operator be given through a specific realization of the corresponding Poisson algebra [29]. But then, it is not known how to connect this approach with that of the Bäcklund matrix. The two approaches, although linked to the same ideas, could not be unified easily. In the first picture, one would like to deduce the Poisson brackets of the defect matrix from its interpretation as a Bäcklund matrix, but this is hindered by the divergence of the Poisson brackets at coinciding space points. In the second picture, the form of the Poisson brackets for the defect matrix is postulated a priori using extra local fields, but it then becomes difficult to eliminate those extra fields. In spite of the significant success of these investigations, this issue is still open and represents a wide gap in the understanding of Liouville integrability for theories with a defect.

The purpose of this paper is to bridge this gap by reconciling these two points of view, and at the same time to get rid of the limitations of each approach by proposing a significantly new idea. Our approach also allows for a natural reinterpretation of the defect density in the Lagrangian picture. Therefore, it provides a unifying framework of the three different approaches used so far for the questions of integrable defects. The idea is to introduce an additional Poisson structure in the theory, in terms of which the defect conditions appear as canonical transformations. This was the missing ingredient in reconciling the various pictures and we show that the method of the classical $r$-matrix and Liouville integrability with respect to the new Poisson structure follows trivially from this observation. Interestingly, this new Poisson structure, which we call equal-space 
Poisson bracket, has been known for a long time in other areas under the generic name of multisymplectic formalism. Although this research area has developed in a rather non systematic way (see e.g. [32] for an attempt to give an account of the various approaches) and into a heavy mathematical formalism, the commonly accepted origin is the so-called De Donder-Weyl formalism [33, 34]. The basic observation is that the traditional canonical formalism (either classical or quantum) is grossly unbalanced in the way it treats time as opposed to the other coordinates. The De Donder-Weyl formalism, also called covariant field theory formalism, aims at treating all independent variables on the same footing. We keep this simple idea and implement it directly in the context of our defect problem, on the example of the NLS equation, showing how it combines nicely with the method of the classical $r$-matrix.

The organization of the paper is as follows. In the next section, we summarize the motivation for introducing a multisymplectic structure in field theory and introduce the relevant Poisson brackets for our purpose. It is shown how the usual classical r-matrix approach fits in the new approach and we emphasize how the new structure brings in a completely equivalent description for a system without a defect. In section 3, we recall briefly the approach of [25] and then go on to show how the new Poisson structure can be used to discuss Liouville integrability and the classical $r$-matrix approach for the NLS model with a defect. The last section contains our conclusions and perspectives on future directions.

\section{Multisymplectic structure of the NLS equation}

\subsection{Space and time Hamiltonian forms for NLS}

Here we present a systematic account of the multisymplectic structure of the NLS equation by introducing two Poisson brackets on the phase space of the model. This formalises the idea of "dual picture" discussed in [35]. The main observation behind the multisymplectic approach to field theory is that the canonical quantization procedure puts emphasis only on the time parameter and, as a consequence, considers only a partial Legendre transformation when defining canonical conjugate coordinates. The traditional approach goes as follows. Given fields $\phi_{a}$ depending on coordinates $(x, t),{ }^{1}$ one defines the conjugate momenta $\pi^{a}$ as

$$
\pi^{a}=\frac{\partial \mathcal{L}}{\partial\left(\partial_{t} \phi_{a}\right)},
$$

$\mathcal{L}$ being the Lagrangian density. Then, one imposes equal-time canonical relations by defining the space Poisson brackets as

$$
\left\{\phi_{a}\left(x, t_{0}\right), \pi^{b}\left(y, t_{0}\right)\right\}_{S}=\delta_{a}^{b} \delta(x-y),
$$

at some initial time $t_{0}$, with the other brackets being trivial. The subcript $S$ indicates that the Poisson bracket is of equal-time i.e. it does not depend on time but only on the space

\footnotetext{
${ }^{1}$ For simplicity here, we only consider two coordinates as this is enough for our purposes in $1+1$ dimensional field theory.
} 
variables. However, the Legendre transformation (2.1) is in fact incomplete, since one can define also another complimentary set of conjugate momenta as

$$
\Pi^{a}=\frac{\partial \mathcal{L}}{\partial\left(\partial_{x} \phi_{a}\right)} .
$$

The second "dual" Poisson bracket is then defined in complete analogy by

$$
\left\{\phi_{a}\left(x_{0}, t\right), \Pi^{b}\left(x_{0}, \tau\right)\right\}_{T}=\delta_{a}^{b} \delta(t-\tau),
$$

at some fixed location $x_{0}$, with the other brackets being zero. These relations may be seen as equal-space canonical brackets. The subscript $T$ indicates that this Poisson bracket does not involve space variables. These two brackets can be combined and form the basis of the formulation of covariant Poisson brackets for field theories.

We now discuss this idea in detail for the NLS and show that the two brackets provide an equivalent description of the model. Moreover, this setting puts the two components of the Lax pair on an equal footing and the coexistence of the two brackets is totally compatible with the usual properties of NLS model, like the existence of an infinite number of conserved quantities. The main result of this section is, that the method of the classical $r$ matrix, developed only for the bracket $\{,\}_{S}$, and based on the space part of the Lax pair, goes over entirely to the new picture where one uses $\{,\}_{T}$ and the time part of the Lax pair.

We emphasize strongly, that the multisymplectic formalism presented here is different from the well-known bi-Hamiltonian theory of integrable systems [36]. The bi-Hamiltonian theory is based on the existence of two compatible equal-time brackets $\{,\}_{S 1}$ and $\{,\}_{S 2}$, each of which allows for the description of the time evolution of the model. Our equal-space bracket $\{,\}_{T}$ on the other hand is linked to the space evolution of the model. It is based on a completely different Legendre transformation that is not considered in the traditional canonical approach.

Consider the NLS equation

$$
i q_{t}+q_{x x}=2 \epsilon|q|^{2} q, \quad \epsilon= \pm 1,
$$

for the complex field $q(x, t)$. One can view the real and imaginary parts of $q$ as the fields of the theory or equivalently can take $q \equiv \phi_{1}$ and its complex conjugate $q^{*} \equiv \phi_{2}$ as two independent fields. A Lagrangian density for this equation is

$$
\mathcal{L}=\frac{i}{2}\left(\phi_{2} \phi_{1 t}-\phi_{2 t} \phi_{1}\right)-\phi_{2 x} \phi_{1 x}-\epsilon\left(\phi_{2} \phi_{1}\right)^{2}
$$

From this, we get

$$
\pi^{1}=\frac{i}{2} \phi_{2}, \quad \pi^{2}=-\frac{i}{2} \phi_{1},
$$

One then obtains the NLS equation consistently ${ }^{2}$ as

$$
\pi_{t}^{j}=\frac{1}{2}\left\{\pi^{j}, H_{S}\right\}_{S}, \quad j=1,2,
$$

\footnotetext{
${ }^{2}$ There is a subtlety here related to the fact that the Lagrangian for NLS is linear in the "velocities" $\phi_{j t}$. Accordingly, one has to use the Dirac bracket. In our case, the net result of the standard approach to constrained systems is the factor $\frac{1}{2}$ in front of the bracket.
} 
where $H_{S}=\int \mathcal{H}_{S} d x$ and

$$
\mathcal{H}_{S}=\pi^{1} \phi_{1 t}+\pi^{2} \phi_{2 t}-\mathcal{L}=\phi_{1 x} \phi_{2 x}+\epsilon \phi_{1}^{2} \phi_{2}^{2}
$$

The usual presentation of the Hamiltonian formulation of NLS takes a slight shortcut and considers the following canonical Poisson brackets ${ }^{3}$

$\left\{q\left(x, t_{0}\right), q^{*}\left(y, t_{0}\right)\right\}_{S}=-i \delta(x-y), \quad\left\{q\left(x, t_{0}\right), q\left(y, t_{0}\right)\right\}_{S}=0 \quad, \quad\left\{q^{*}\left(x, t_{0}\right), q^{*}\left(y, t_{0}\right)\right\}_{S}=0$.

together with the Hamiltonian density

$$
\mathcal{H}_{S}=\left|q_{x}\right|^{2}+\epsilon|q|^{4}
$$

The equations of motion then read

$$
q_{t}=\left\{q, H_{S}\right\}_{S}
$$

Now, in view of the above discussion on the equal-space canonical brackets, we introduce new canonical conjugate fields to $\phi_{j}$ by

$$
\Pi^{1}=-\phi_{2 x}, \quad \Pi^{2}=-\phi_{1 x},
$$

which leads us to define the following new equal space Poisson brackets for NLS

$$
\begin{aligned}
& \left\{q\left(x_{0}, t\right), q_{x}^{*}\left(x_{0}, \tau\right)\right\}_{T}=-\delta(t-\tau), \quad\left\{q\left(x_{0}, t\right), q^{*}\left(x_{0}, \tau\right)\right\}_{T}=0=\left\{q_{x}\left(x_{0}, t\right), q_{x}\left(x_{0}, \tau\right)\right\}_{T} \\
& \left\{q_{x}\left(x_{0}, t\right), q^{*}\left(x_{0}, \tau\right)\right\}_{T}=\delta(t-\tau), \quad\left\{q\left(x_{0}, t\right), q\left(x_{0}, \tau\right)\right\}_{T}=0=\left\{q_{x}\left(x_{0}, t\right), q\left(x_{0}, \tau\right)\right\}_{T},
\end{aligned}
$$

One then obtains the NLS equation from

$$
\Pi_{x}^{j}=\left\{\Pi^{j}, H_{T}\right\}_{T}, \quad j=1,2,
$$

where $H_{T}=\int \mathcal{H}_{T} d t$ and,

$$
\mathcal{H}_{T}=\Pi^{1} \phi_{1 x}+\Pi^{2} \phi_{2 x}-\mathcal{L}=-\left|q_{x}\right|^{2}-\frac{i}{2}\left(q^{*} q_{t}-q_{t}^{*} q\right)+\epsilon|q|^{4} .
$$

The new Hamiltonian density $\mathcal{H}_{T}$ is the analog of $\mathcal{H}_{S}$ with respect to the new Poisson brackets $\{,\}_{T}$. Indeed, a direct calculation shows that the NLS equation (2.5) is obtained from the Hamiltonian equation (2.15) with $j=2$ as

$$
\begin{aligned}
-q_{x x} & =\int\left\{-q_{x}(t),\left(-\left|q_{x}(\tau)\right|^{2}-\frac{i}{2}\left(q^{*}(\tau) q_{\tau}(\tau)-q_{\tau}^{*}(\tau) q(\tau)\right)+\epsilon|q(\tau)|^{4}\right)\right\} d \tau \\
& =i q_{t}-2 \epsilon|q|^{2} q .
\end{aligned}
$$

\footnotetext{
${ }^{3}$ The reader will note the absence of a factor 2 to compensate for the direct use of $\{,\}_{S}$ instead of the Dirac brackets.
} 


\subsection{Classical $r$-matrix approach for the two Poisson brackets}

\subsubsection{The standard approach with $\{,\}_{S}$}

The NLS equation arises as the compatibility condition of the auxiliary problem

$$
\begin{aligned}
& \Psi_{x}(x, t, \lambda)=U(x, t, \lambda) \Psi(x, t, \lambda), \\
& \Psi_{t}(x, t, \lambda)=V(x, t, \lambda) \Psi(x, t, \lambda),
\end{aligned}
$$

with Lax pair

$$
U=\left(\begin{array}{cc}
-i \lambda & q \\
\epsilon q^{*} & i \lambda
\end{array}\right), \quad V=\left(\begin{array}{cc}
-2 i \lambda^{2}-i \epsilon|q|^{2} & 2 \lambda q+i q_{x} \\
\epsilon\left(2 \lambda q^{*}-i q_{x}^{*}\right) & 2 i \lambda^{2}+i \epsilon|q|^{2}
\end{array}\right) .
$$

The compatibility condition $\Psi_{x t}=\Psi_{t x}$ results in the so-called zero curvature representation

$$
U_{t}-V_{x}+[U, V]=0,
$$

which must hold identically for arbitrary spectral parameter $\lambda$.

Using $U$ and $\{,\}_{S}$, one can develop the standard classical $r$-matrix approach [37-39] to discuss the Liouville integrability of the model. This approach is based entirely on the $x$-part of the Lax pair, eq. (2.19), and time is considered as a fixed parameter, say $t=0$, which is eventually evolved. Therefore, we drop it in this section. The starting point of the method is the following ultralocal Poisson bracket relation that may be derived using the PB structure (2.10):

$$
\left\{U_{1}(x, \lambda), U_{2}(y, \mu)\right\}_{S}=\delta(x-y)\left[r(\lambda-\mu), U_{1}(x, \lambda)+U_{2}(y, \mu)\right]
$$

where we have used the notation $U_{1}=U \otimes \mathbb{I}, U_{2}=\mathbb{I} \otimes U$ and $r(\lambda)$ is the usual $s l_{2}$ classical $r$-matrix

$$
r(\lambda)=\frac{-\epsilon P}{2 \lambda},
$$

$P$ being the permutation operator on $\mathbb{C}^{2} \otimes \mathbb{C}^{2}: P u \otimes v=v \otimes u$. We introduce the monodromy matrix $M_{S}(x, \lambda)$ as the fundamental solution of (2.19) (at $t=0$ ) equal to the identity matrix at $x=0$. Then, one computes for $x>0$, (see e.g. [39])

$$
\left\{M_{S 1}(x, \lambda) \otimes, M_{S 2}(x, \mu)\right\}_{S}=\left[r(\lambda-\mu), M_{S}(x, \lambda) \otimes M_{S}(x, \mu)\right] .
$$

On a finite interval $[0, L]$ with periodic boundary conditions, this relation is enough to conclude about the Liouville integrability of the model in the following sense: the transfer function $\mathcal{T}_{S}(\lambda)=\operatorname{tr} M_{S}(L, \lambda)$ commutes for different spectral parameters

$$
\left\{\mathcal{T}_{S}(\lambda), \mathcal{T}_{S}(\mu)\right\}=0
$$

due to (2.25), and hence generates the infinite set of conserved quantities $I_{n}, n \in \mathbb{N}$, which are in involution with respect to $\{,\}_{S}$

$$
\left\{I_{n}, I_{m}\right\}_{S}=0, \quad n, m \in \mathbb{N}
$$


These quantities can be extracted as local functionals of the fields $q$ and $q^{*}$ algorithmically by studying the large (real) $\lambda$ expansion of the transfer function. Full details are given in section I.4 of [39] and the net results is that, as $|\lambda| \rightarrow \infty$,

$$
\arccos \left(\frac{1}{2} \mathcal{T}_{S}(\lambda)\right)+\lambda L=\epsilon \sum_{n=1}^{\infty} \frac{I_{n}}{\lambda^{n}},
$$

where the integrals of motion $I_{n}$ are given by

$$
I_{n}=\int_{0}^{L} q^{*}(x, t) w_{n}(x, t) d x
$$

and determined recursively using

$$
w_{1}=q, \quad w_{n+1}=-i \frac{\partial w_{n}}{\partial x}+\epsilon q^{*} \sum_{k=1}^{n-1} w_{k} w_{n-k} .
$$

In particular, using $I_{3}$ (and an integration by parts) one extracts the Hamiltonian $H_{S}$ precisely as

$$
H_{S}=\int_{0}^{L}\left(\left|q_{x}\right|^{2}+\epsilon|q|^{4}\right) d x=\int_{0}^{L} \mathcal{H}_{S} d x
$$

where $\mathcal{H}_{S}$ is the density given in (2.11). For our purposes, it is more convenient to arrive at the same result directly from the Lax pair formulation. Representing $\Psi$ in (2.19), (2.20) as a column vector

$$
\Psi=\left(\begin{array}{c}
\Psi^{1} \\
\Psi^{2}
\end{array}\right)
$$

and denoting $\Gamma=\Psi^{2}\left(\Psi^{1}\right)^{-1}$, we derive

$$
\begin{aligned}
& \left(\ln \Psi^{1}\right)_{x}=U^{11}+U^{12} \Gamma, \\
& \left(\ln \Psi^{1}\right)_{t}=V^{11}+V^{12} \Gamma,
\end{aligned}
$$

where $U^{i j}, V^{i j}$ are the entries of $U$ and $V$. Then, $\left(\ln \Psi^{1}\right)_{x t}=\left(\ln \Psi^{1}\right)_{t x}$ yields the conservation equation

$$
\left(U^{12} \Gamma\right)_{t}=\left(V^{11}+V^{12} \Gamma\right)_{x},
$$

since $U^{11}$ is constant. This shows using the periodic boundary condition, that $\int_{0}^{L} U^{12} \Gamma d x$ is a generating function of the conserved quantities. One can also derive a Riccati equation for $\Gamma$

$$
\Gamma_{x}=2 i \lambda \Gamma+\epsilon q^{*}-q \Gamma^{2},
$$

by using (2.19). Expanding $\Gamma$ at $\lambda \rightarrow \infty$ :

$$
\Gamma=\epsilon \sum_{n=1}^{\infty} \frac{\Gamma_{n}}{(2 i \lambda)^{n}},
$$


and inserting in the Riccati equation, one gets a recursion relation for $\Gamma_{n}$

$$
\Gamma_{1}=q^{*}, \quad \Gamma_{n+1}=\Gamma_{n x}+\epsilon q \sum_{k=1}^{n-1} \Gamma_{k} \Gamma_{n-k}, \quad n \geq 1
$$

which allows to determine the successive integrals of motion $J_{n}$ as local functionals of the field and its space derivatives

$$
J_{n}=\int_{0}^{L} q \Gamma_{n} d x, \quad n \geq 1 .
$$

Note that $I_{n}^{*}=(i)^{n-1} J_{n}$ and one usually uses the combination $\frac{1}{2}\left(I_{n}+I_{n}^{*}\right)$ to get realvalued conserved quantities. The connection just discussed between the $I_{n}$ and $J_{n}$ shows that the conserved quantities $J_{n}$ derived directly from the Lax pair presentation are in involution with respect to the Poisson structure $\{,\}_{S}$ introduced to describe NLS as a Hamiltonian system.

\subsubsection{Classical $r$-matrix approach for the new bracket $\{,\}_{T}$}

In this section, we show that one can formulate a treatment for the $t$-part of the auxiliary problem (2.20), that goes completely parallel to the usual classical $r$-matrix approach discuss above. One has to use the new Poisson bracket $\{,\}_{T}$ and the starting point is now an ultralocal relation involving the time Lax matrix $V$. The variable $x$ is a fixed parameter, say $x=x_{0}$, which could evolve eventually. We have the following

Proposition 2.1. Let the Poisson bracket $\{,\}_{T}$ be given by (2.14) and $V$ be given by (2.21). Then,

$$
\left\{V_{1}(t, \lambda), V_{2}(\tau, \mu)\right\}_{T}=-\delta(t-\tau)\left[r(\lambda-\mu), V_{1}(t, \lambda)+V_{2}(\tau, \mu)\right],
$$

with the same classical r-matrix as in (2.24).

Proof. The proof is essentially the same as for $U$ and follows from direct computation. We only give the main steps to illustrate the differences with the usual computation. Denote $U=-i \lambda \sigma_{3}+W, W=q \sigma^{+}+\epsilon q^{*} \sigma^{-}$and then note that

$$
V=-2 i \lambda^{2} \sigma_{3}+2 \lambda W-i \sigma_{3} W_{x}-i W^{2} \sigma_{3} .
$$

In view of (2.14), the Poisson brackets $\left\{V_{1}(t, \lambda) \otimes V_{2}(\tau, \mu)\right\}$ only involves four terms

$$
\begin{aligned}
\left\{V_{1}(t, \lambda) \otimes V_{2}(\tau, \mu)\right\}= & -2 i \lambda\left\{W_{1}(t), W_{x 2}(\tau)\right\}\left(\mathbb{I} \otimes \sigma_{3}\right)-2 i \mu\left\{W_{x 1}(t), W_{2}(\tau)\right\}\left(\sigma_{3} \otimes \mathbb{I}\right) \\
& -\left\{W_{1}^{2}(t), W_{x 2}(\tau)\right\}\left(\sigma_{3} \otimes \sigma_{3}\right)-\left\{W_{x 1}(t), W_{2}^{2}(\tau)\right\}\left(\sigma_{3} \otimes \sigma_{3}\right) .
\end{aligned}
$$

Performing some algebra yields

$$
\begin{aligned}
\left\{V_{1}(t, \lambda) \otimes V_{2}(\tau, \mu)\right\}=\epsilon \delta(t-\tau)\left[2 i ( \lambda + \mu ) \left(\sigma_{+} \otimes \sigma_{-}-\right.\right. & \left.\sigma_{-} \otimes \sigma_{+}\right) \\
& \left.+(\mathbb{I} \otimes W-W \otimes \mathbb{I})\left(\sigma_{3} \otimes \sigma_{3}\right)\right] .
\end{aligned}
$$


On the other hand,

$$
\left[P, V_{1}(t, \lambda)+V_{2}(t, \mu)\right]=2(\lambda-\mu)\left[i(\lambda+\mu)\left(\sigma_{3} \otimes \mathbb{I}-\mathbb{I} \otimes \sigma_{3}\right)+(\mathbb{I} \otimes W-W \otimes \mathbb{I})\right] P .
$$

Noting that $\left(\sigma_{3} \otimes \mathbb{I}-\mathbb{I} \otimes \sigma_{3}\right) P=2\left(\sigma_{+} \otimes \sigma_{-}-\sigma_{-} \otimes \sigma_{+}\right)$and $(\mathbb{I} \otimes W-W \otimes \mathbb{I}) P=$ $(\mathbb{I} \otimes W-W \otimes \mathbb{I})\left(\sigma_{3} \otimes \sigma_{3}\right)$ and using the expression (2.24) for the $r$-matrix, we get the result (see also [35]).

As a direct consequence, we obtain

Corollary 2.2. Let $M_{T}(t, \lambda)$ be the fundamental solution of (2.20) (at $x=x_{0}$ ) satisfying $M_{T}(0, \lambda)=\mathbb{1}$, then for $t>0$,

$$
\left\{M_{T 1}(t, \lambda), M_{T 2}(t, \mu)\right\}_{T}=-\left[r(\lambda-\mu), M_{T}(t, \lambda) \otimes M_{T}(t, \mu)\right] .
$$

If we work on a finite time interval $[0, \tau]$ with periodic conditions in time $q(x, 0)=$ $q(x, \tau)$, we deduce that the transfer function $\mathcal{T}_{T}(\lambda)=\operatorname{tr} M_{T}(\tau, \lambda)$, Poisson commutes for different values of the spectral parameter. We can therefore talk about Liouville integrability of NLS in the same sense as before but viewed with respect to $\{,\}_{T}$. The transfer function $\mathcal{T}_{T}(\lambda)$ generates the conserved quantities (in space now) which are in involution with respect to $\{,\}_{T}$. To extract these conserved quantities, we follow the same reasoning as in the previous section and use the conservation equation (2.35). But this time, we interpret it differently, that is, as showing that $\int_{0}^{\tau}\left(V^{11}+V^{12} \Gamma\right) d t$ is a generating function for the conserved quantities in space. Combined with the following time-Riccati equation for $\Gamma$

$$
\Gamma_{t}=V^{21}+\left(V^{22}-V^{11}\right) \Gamma-V^{12} \Gamma^{2}
$$

we obtain a complete analog of the above algorithm for computing recursively the conserved quantities. Inserting the expansion

$$
\Gamma=\epsilon \sum_{n=1}^{\infty} \frac{\gamma_{n}}{(2 i \lambda)^{n}},
$$

we obtain

$$
\begin{aligned}
\gamma_{1} & =-q^{*}, \quad \gamma_{2}=-q_{x}^{*}, \quad \gamma_{3}=-i q_{t}^{*}-\epsilon q^{* 2} q \\
\gamma_{n+2} & =i \gamma_{n t}+2 \epsilon|q|^{2} \gamma_{n}+\epsilon q \sum_{k=1}^{n} \gamma_{k} \gamma_{n+1-k}-\epsilon q_{x} \sum_{k=1}^{n-1} \gamma_{k} \gamma_{n-k}, \quad n \geq 1 .
\end{aligned}
$$

Writing $V^{11}+V^{12} \Gamma=-2 i \lambda^{2}+\epsilon \sum_{n=1}^{\infty} \frac{\mathcal{K}_{n}}{(2 i \lambda)^{n}}$, the corresponding integrals are

$$
K_{n}=\int_{0}^{\tau} \mathcal{K}_{n} d t=\int_{0}^{\tau} i\left(q_{x} \gamma_{n}-q \gamma_{n+1}\right) d t
$$

They are in involution for the new Poisson bracket

$$
\left\{K_{n}, K_{m}\right\}_{T}=0, \quad n, m \in \mathbb{N} .
$$


In particular, we find that

$$
\mathcal{H}_{T}=\frac{i}{2}\left(\mathcal{K}_{2}^{*}-\mathcal{K}_{2}\right)
$$

where $\mathcal{H}_{T}$ is the Hamiltonian density given in (2.16). Hence, we recover the Hamiltonian $H_{T}$ precisely as

$$
H_{T}=\int_{0}^{\tau} \mathcal{H}_{T} d t=\int_{0}^{\tau}\left(-q_{x}^{*} q_{x}-\frac{i}{2}\left(q^{*} q_{t}-q q_{t}^{*}\right)+\epsilon\left(q^{*} q\right)^{2}\right) d t .
$$

This concludes our presentation of the multisymplectic approach to NLS.

Remark: the choice of periodic boundary conditions in time is solely motivated by the need to keep the discussion as concise as possible at the technical level. Of course, by analogy with the usual "space" case, one could consider "open" boundary conditions at $t=0$ and $t=\tau$. In that case, the analog of Sklyanin's theory for systems on an interval should be implemented. This can obviously be done here since the fundamental algebraic structure is the same. Another possibility would be to consider vanishing conditions at infinity. Again, there is no deep obstacle to this. The usual class of solutions obtained from initial conditions satisfying $\lim _{|x| \rightarrow \infty} q(x, 0)=0$ contains for instance the well-known $N$-soliton solutions. These solutions are in fact well-defined for all $t \in \mathbb{R}$ and satisfy $\lim _{|t| \rightarrow \infty} q\left(x_{0}, t\right)=0$ for arbitrary but fixed $x_{0}$. Therefore, it would make sense to consider the time problem on the line with vanishing boundary conditions at infinity.

\subsection{Canonical transformations and Bäcklund transformations}

We recall some elementary facts about canonical transformations in the Hamiltonian formalism. The main message from sections 2.1 and 2.2 is that there is a complete duality in the structures and the Hamiltonian formalism for NLS whether one uses the (usual) space point of view or the (new) time point of view. Therefore, all we have to do to discuss canonical transformations for NLS simultaneously for $\{,\}_{S}$ and $\{,\}_{T}$ is to use a generic Poisson bracket $\{,\}_{Z}$ and canonical fields $Q_{j}, P^{j}$ of the independent variables $u, v$. The results will apply to each situation simply by performing the following identifications:

- Traditional approach:

$$
\{,\}_{Z}=\{,\}_{S}, Q_{j}=\phi_{j}, P^{j}=\pi^{j}, u=x, v=t \text { (with } t \text { fixed) },
$$

- New approach:

$$
\left.\{,\}_{Z}=\{,\}_{T}, Q_{j}=\phi_{j}, P^{j}=\Pi^{j}, u=t, v=x \text { (with } x \text { fixed }\right) .
$$

Given our purposes below in connection with canonical properties of Bäcklund transformations, we follow the method of $[40,41]$ generalizing it to our new approach and consider canonical transformations $\left\{Q_{j}, P_{j}\right\} \rightarrow\left\{\widetilde{Q}_{j}, \widetilde{P}_{j}\right\}$ that preserve the form of the local conserved densities $\mathcal{I}_{n}$ of the theory, in the sense that there should exists functionals $\mathcal{F}_{n}$ such that

$$
\widetilde{\mathcal{I}}_{n}=\mathcal{I}_{n}+\partial_{u} \mathcal{F}_{n}
$$


For the conserved quantities, this yields

$$
\widetilde{I}_{n}=I_{n}+E_{n}
$$

where $E_{n}$ are constants obtained by integrating $\partial_{u} \mathcal{F}_{n}$ on the relevant interval $U$ for $u$. Of course, in the traditional approach where $u=x$ and $U$ is either $\mathbb{R}$ (with vanishing conditions for the fields and their derivatives at infinity) or the interval $[0, L]$ (with periodic boundary conditions), these constants are zero. This is a natural generalization to the case of integrable systems of the usual notion of canonical transformations that are required to preserve the form of the Hamiltonian

$$
\widetilde{H}=H+E,
$$

where the constant $E$ comes from the fact that one considers so-called restricted canonical transformations. More precisely, one requires that the one-forms representing the system in old and new variables differ only by a exact form

$$
\int_{U} d u\left(\widetilde{P}^{j} d \widetilde{Q}_{j}\right)-\widetilde{H} d v=\int_{U} d u\left(P^{j} d Q_{j}\right)-H d v+d F
$$

where $F$ is the so-called generating functional and is taken to be as

$$
F\left[Q_{j}, P^{j}, \widetilde{Q}_{j}, \widetilde{P}^{j}, v\right]=S\left[Q_{j}, P^{j}, \widetilde{Q}_{j}, \widetilde{P}^{j}\right]-E v .
$$

Assuming that the new variables do not depend explicitely on $v$, we get the well-known transformation formulas

$$
P^{j}=\frac{\delta F}{\delta Q_{j}}, \quad \widetilde{P}^{j}=-\frac{\delta F}{\delta \widetilde{Q}_{j}},
$$

where we have assumed that $Q_{j}$ and $\widetilde{Q}_{j}$ were functionally independent variables (corresponding to the so-called type 1 generating functional).

In the traditional approach (2.50), the above discussion, in particular eq. (2.52), was used in $[40,41]$ to show that Bäcklund transformations naturally arise as canonical transformations of the restricted type considered here. For clarity and self-containedness, we rewrite here the main line of arguments but expressed directly in the Lax pair formalism. Since we want to preserve the form of the Hamiltonian (and hence the equation of motion) as well as that of all conserved quantities, we look for a transformation that preserves the zero curvature representation of the equation of motion. Looking at the $x$ part only of the auxiliary problem (2.19), for fixed $t$, we introduce a matrix $L$ such that $\widetilde{\Psi}(x, \lambda)=L(x, \lambda) \Psi(x, \lambda)$, and satisfying

$$
\partial_{x} L=\widetilde{U} L-L U,
$$

where $\widetilde{U}$ is of the same form as $U$ but with $\phi_{j}$ replaced with $\widetilde{\phi_{j}}$. As explained in section 2.2.1, the infinite set of conserved quantities is generated by

$$
J(\lambda)=\int_{0}^{L} U^{12} \Gamma(\lambda) d x
$$


and similarly for the conserved quantities in the new variables

$$
\widetilde{J}(\lambda)=\int_{0}^{L} \widetilde{U}^{12} \widetilde{\Gamma}(\lambda) d x,
$$

with obvious notations. Therefore, $(2.52)$ will be fulfilled if we can write

$$
\widetilde{U}^{12} \widetilde{\Gamma}(\lambda)=U^{12} \Gamma(\lambda)+\partial_{x} \mathcal{F}(\lambda),
$$

for some functional $\mathcal{F}$. Now using the definition for $L,(2.58)$ and the Riccati equation (2.36), we find

$$
\widetilde{U}^{12} \widetilde{\Gamma}(\lambda)=U^{12} \Gamma(\lambda)+\partial_{x} \ln \left(L^{11}+L^{12} \Gamma(\lambda)\right) .
$$

hence $\mathcal{F}=\ln \left(L^{11}+L^{12} \Gamma\right)$, and the Bäcklund transformation associated to $L$ is canonical. A consequence of this result is that the Lax matrix $\widetilde{U}(x, \lambda)$ also satisfies the ultralocal relations (2.23). Indeed, a standard argument shows that the new variables $\widetilde{\phi}_{j}$ and $\widetilde{\pi}_{j}$ satisfy the same canonical Poisson brackets as $\phi_{j}$ and $\pi_{j}$, i.e. (2.10) in the present case. The method of the classical $r$-matrix can then be used entirely for the new variables $\widetilde{\phi}_{j}$ and $\widetilde{\pi}_{j}$.

The adaptation of this reasoning to the new approach (2.51), done in the next section, is the key in reinterpreting defect conditions arising from frozen Bäcklund transformations at a fixed location $x=x_{0}$ as canonical transformations of the system. As a consequence, we will be able to conclude on the Liouville integrability of NLS with such defect conditions.

\section{$3 \quad$ NLS with a defect: Liouville integrability}

\subsection{Defects as frozen Bäcklund transformations}

Viewing a defect in space as an internal boundary condition on the fields and their time and space derivatives at a given point, the fruitful idea of frozen Bäcklund transformations, originally noticed in $[18,19]$, is a convenient way of introducing integrable defects in classical field theories described by a Lax pair. The systematic procedure for a large class of integrable classical field theories was described and implemented in [25], where a generating function for the defect contributions to the conserved quantities was explicitely constructed. This allows to speak of the integrability of such defect conditions in the sense of the existence of an infinite number of conserved quantities.

The main steps go as follows. Consider another copy of the auxiliary problem for $\widetilde{\Psi}$ with Lax pair $\widetilde{U}, \widetilde{V}$ defined as in (2.19), (2.20) with the new field $\widetilde{q}$ replacing $q$. We fix a point $x_{0} \in[0, L]$ and use the auxiliary problem (2.19), (2.20) to describe the system $x>x_{0}$, while the one with $\widetilde{U}$ and $\widetilde{V}$ describe the system for $x<x_{0}$. At $x=x_{0}$, the two systems are connected via the condition

$$
\widetilde{\Psi}\left(x_{0}, t, \lambda\right)=L_{0}(t, \lambda) \Psi\left(x_{0}, t, \lambda\right) .
$$

In turn, this yields the defect conditions in the form

$$
L_{0 t}(t, \lambda)=\widetilde{V}\left(x_{0}, t, \lambda\right) L_{0}(t, \lambda)-L_{0}(t, \lambda) V\left(x_{0}, t, \lambda\right),
$$


where we have denoted $V\left(x_{0}, t, \lambda\right)=\lim _{x \rightarrow x_{0}} V(x, t, \lambda)$ and similarly for $\widetilde{V}$. The matrix $L$ is called the defect matrix. With this construction, one can identify the generating function of the defect contribution to the conserved quantities as follows [25].

Proposition 3.1. The generating function for the integrals of motion reads

$$
I(\lambda)=I_{\mathrm{bulk}}^{\mathrm{left}}(\lambda)+I_{\mathrm{bulk}}^{\mathrm{right}}(\lambda)+I_{\mathrm{defect}}(\lambda),
$$

where

$$
\begin{aligned}
I_{\text {bulk }}^{\text {left }}(\lambda) & =\int_{0}^{x_{0}} \widetilde{U}^{12} \widetilde{\Gamma} d x, \quad I_{\text {bulk }}^{\text {right }}(\lambda)=\int_{x_{0}}^{L} U^{12} \Gamma d x, \\
I_{\text {defect }}(\lambda) & =\left.\ln \left(L^{11}+L^{12} \Gamma\right)\right|_{x=x_{0}},
\end{aligned}
$$

and $L^{i j}$ 's are the entries of the defect matrix $L$. This means that

$$
\partial_{t} I(\lambda)=0
$$

The previous result gives the generating function of the infinite set of modified conserved quantities (in time) and can be combined with (2.38) and (2.39) to extract them order by order. From the point of view of PDEs, one can then speak of integrability, though from the point of view of Hamiltonian integrable systems, the question of Liouville integrability is still not solved. This is done in the next section.

\subsection{Liouville integrability: defect conditions as canonical transformations}

The discussion of section 2.3 and the short review in the previous section about defect conditions arising from frozen Bäcklund transformations make it plain that such defect conditions are nothing but canonical transformations for the new bracket $\{,\}_{T}$. Indeed, one can repeat word for word the arguments of section 2.3 but using the new approach (2.51) instead of the usual one (2.50). The key equations (2.58) and (2.62) are replaced respectively by (3.2) and

$$
\widetilde{V}^{11}+\widetilde{V}^{12} \widetilde{\Gamma}=V^{11}+V^{12} \Gamma+\partial_{t} \ln \left(L_{0}^{11}+L_{0}^{12} \Gamma\right)
$$

Here, the time Riccati equation (2.42) should be used in establishing this last result. Comparing (3.7) with the general discussion of section 2.3, we see that, denoting $\mathcal{E}_{2}$ as the coefficient of $\lambda^{-2}$ in the expansion of $i\left(\ln \left(L_{0}^{11}+L_{0}^{12} \Gamma\right)^{*}-\ln \left(L_{0}^{11}+L_{0}^{12} \Gamma\right)\right)$, we obtain

$$
\widetilde{H}_{T}=H_{T}+\left[\mathcal{E}_{2}\right]_{0}^{\tau}
$$

The canonical transformation formulas (2.57) also allow us to reinterpret the defect lagrangian density originally introduced in the Lagrangian approach to integrable defects $[18$, 19] as the density for the generating functional of the canonical transformation. This provides an explicit check that the frozen Bäcklund defect conditions are indeed canonical 
transformations with respect to our Poisson structure. For instance, from the defect density given in [42] (eq. (3.2)), in the focusing case $\epsilon=-1$, we find that by choosing $S$ in $(2.56)$ as

$$
\begin{array}{r}
S\left[\phi_{j}, \widetilde{\phi}_{j}, \Pi^{j}, \widetilde{\Pi}^{j}\right]=\int_{0}^{\tau}\left(\frac{i \Omega}{2} \partial_{t} \ln \left(\frac{\widetilde{\phi}_{1}-\phi_{1}}{\widetilde{\phi}_{2}-\phi_{2}}\right)+\frac{\Omega^{3}}{3}\right. \\
\left.+\Omega\left(\widetilde{\phi}_{1} \widetilde{\phi}_{2}+\phi_{1} \phi_{2}-\alpha^{2}\right)-i \alpha\left(\widetilde{\phi}_{2} \phi_{1}-\widetilde{\phi}_{1} \phi_{2}\right)\right) d t
\end{array}
$$

where

$$
\Omega= \pm \sqrt{\beta^{2}-\left(\widetilde{\phi}_{1}-\phi_{1}\right)\left(\widetilde{\phi}_{2}-\phi_{2}\right)}
$$

then, after some algebra, eqs (2.57) yield the following defect conditions at $x=x_{0}$

$$
\left\{\begin{array}{l}
\widetilde{\phi}_{1 x}-\phi_{1 x}=i \alpha\left(\widetilde{\phi}_{1}-\phi_{1}\right)+\left(\widetilde{\phi}_{1}+\phi_{1}\right) \Omega, \\
\widetilde{\phi}_{1 t}-\phi_{1 t}=-\alpha\left(\widetilde{\phi}_{1 x}-\phi_{1 x}\right)+i\left(\widetilde{\phi}_{1 x}+\phi_{1 x}\right) \Omega+i\left(\widetilde{\phi}_{1}-\phi_{1}\right)\left(\widetilde{\phi}_{1} \widetilde{\phi}_{2}+\phi_{1} \phi_{2}\right),
\end{array}\right.
$$

upon recalling that $\widetilde{\Pi}_{2}=-\widetilde{\phi}_{1 x}, \Pi_{2}=-\phi_{1 x}$. These are precisely the defect conditions found in [25], $\alpha$ and $\beta$ being two arbitrary real numbers known to parametrise the Bäcklund transformation for NLS.

At this stage, it is important to analyse what we have just obtained and our claim of Liouville integrability of the NLS model with a defect. The point is that, for $x \in\left[0, x_{0}\right)$, we describe the NLS model in the bulk using the new Poisson bracket $\{,\}_{T}$ and the associated transfer function $\mathcal{T}_{T}(\lambda)$, which ensures that the system is Liouville integrable, as discussed in detail in section 2.2.2. At $x=x_{0}$, we simply reinterpret the defect conditions as a passage from the old canonical variable $\phi_{j}, \Pi_{j}$ (see (2.13) and (2.14)) to new canonical variables $\widetilde{\phi}_{j}, \widetilde{\Pi}_{j}$. From the point of view of the new bracket, the defect conditions are simply a canonical change of variables used to describe the system. The bulk system for $x \in\left(x_{0}, L\right]$ is then the result of the space evolution from $x=x_{0}$ to $x=L$ of the system described in the new variables. Therefore, we simply have to apply the results of section 2.2.2 in the new canonical variables to conclude about the Liouville integrability of the system. In particular, $\widetilde{\mathcal{T}}_{T}(\lambda)$ generates the conserved quantities that are in involution. By construction, the new conserved quantities $\widetilde{K}_{n}$ only differ from the old ones $K_{n}$ by constants (which vanish under our assumptions of periodicity in time). For instance, as seen above at the level of the Hamiltonian, the corresponding constant is $\left[\mathcal{E}_{2}\right]_{0}^{\tau}$. Therefore, at $x=x_{0}$, one is free to use either the old or the new canonical variables to describe the system. The difference between the two canonical pictures is entirely encoded in the defect matrix $L$. This will be even more transparent in the next section when we introduce the monodromy matrix of the system with defect. At the location of the defect, we will have two equivalent options to represent the monodromy matrix (see (3.12) below).

\subsection{Liouville integrability: classical $r$-matrix approach with defect}

Let us first recall the problem that one encounters, when one wants to generalise directly the $r$ matrix approach of $(2.25)$ based on $M_{S}$ and $\{,\}_{S}$, to the case with an integrable 
defect of the type described above. From this point of view, the natural object to consider is the monodromy matrix $\mathcal{M}_{S}(\lambda) \equiv \widetilde{M}_{S}\left(0, x_{0}, \lambda\right) L_{0}(0, \lambda) M_{S}\left(x_{0}, L, \lambda\right)$, where we have added an argument in $\widetilde{M}_{S}$ and $M_{S}$ to specify what part of the interval $[0, L]$ they encode. The problems then come in two flavours. First, there is a serious technical difficulty appearing when one wants to compute the Poisson bracket $\left\{\mathcal{M}_{S}(\lambda), \mathcal{M}_{S}(\mu)\right\}_{S}$ due to the fact that, in this picture, $L_{0}$ is expressed in terms of the fields at coinciding points in space (the location $x_{0}$ of the defect). This can be overcome with great effort thanks to a discretization procedure as described in [28]. But then, there is a conceptual difficulty: one replaces the fields of the bulk evaluated at the location of the defect by new local fields inside $L_{0}$. Then, the desired form of the Poisson bracket involving $L_{0}$ with itself is postulated ad hoc "to make things work", hence imposing the Poisson brackets of the local degrees of freedom living at the defect location. In other words, one simply assumes that $L_{0}$ satisfies the Poisson algebra (2.25). Then, one checks a posteriori, that this is consistent, which gives rise to the "sewing conditions" of [28] between the bulk fields and the defect fields.

As we have argued above, the use of the new Poisson brackets to discuss the Hamiltonian structure of NLS with a defect allows us to reinterpret the defect conditions simply as a canonical transformation, whereby one decides to change the variables used to describe the system at a specific point in space and then lets the system evolve (in space) in the new canonical variables. We now show, that this new point of view allows us to solve the above problems in a natural way. Liouville integrability with a defect, already established in the previous section, is then also manifested through the classical $r$-matrix formalism. In our setting, the natural object to consider is the monodromy matrix $\mathcal{M}_{T}(x, t, \lambda)$ analogous to $M_{T}$ of section 2.2.2, but which takes into account the fact that we change the variables at the location $x=x_{0}$. It is given by

$$
\mathcal{M}_{T}(x, t, \lambda)= \begin{cases}\widetilde{M}_{T}(t, \lambda), & 0 \leq x<x_{0}, \\ \widetilde{M}_{T}(t, \lambda)=L_{0}(t, \lambda) M_{T}(t, \lambda), & x=x_{0}, \\ M_{T}(t, \lambda), & x_{0}<x \leq L .\end{cases}
$$

where $M_{T}$ is the matrix considered in Corollary 2.2 and $\widetilde{M}_{T}$ is the analogous matrix but constructed from the new canonical variables. So we obtain immediately, for all $x \in[0, L]$,

$$
\left\{\mathcal{M}_{T 1}(x, t, \lambda), \mathcal{M}_{T 2}(x, t, \mu)\right\}_{T}=-\left[r(\lambda-\mu), \mathcal{M}_{T 1}(x, t, \lambda) \mathcal{M}_{T 2}(x, t, \mu)\right] .
$$

The transfer matrix of the system with defect is now

$$
\mathcal{T}_{T}^{d}(\lambda)=\operatorname{Tr} \mathcal{M}_{T}(x, \tau, \lambda),
$$

and it generates the conserved quantities which are in involution with respect to $\{,\}_{T}$.

\section{Concluding remarks}

Using the example of NLS, we have introduced the notion of multisymplectic formalism in the context of integrable classical field theory. This was motivated by an unresolved issue in 
the area of integrable defects in classical field theory. We showed the complete equivalence between the usual space canonical approach and new time canonical formulation for NLS equation without a defect. The equivalence goes over to the associated classical r-matrix approaches. The advantage of using the new Poisson structure becomes apparent when one incorporates integrable defects in the form of frozen Bäcklund transformations at a specific location. Indeed, with respect to the new structure, these can be reinterpreted as canonical transformations and one can immediately conclude on the Liouville integrability of the system with such a defect. Once again, this goes over to the classical $r$ matrix formalism where the presence of the defect is absorbed in the time monodromy matrix by changing the canonical variables used to describe the system. This clarifies the missing picture between the Bäcklund approach to integrable defects and the (standard) classical r matrix appraoch.

Let us remark that we have three related interpretations for an integrable defect, each of which having its advantages, that are now unified in our picture. Historically, in the Lagrangian appraoch of $[18,19]$, the defect was introduced as a set of boundary conditions chosen so as to ensure that certain quantities are restored as conserved quantities once the defect contribution is taken into account. The prime example was momentum which is lost a priori due to the breaking of translational symmetry. Then, it was noticed by the same authors that the boundary conditions they found this way were (frozen) Bäcklund transformations. This observation was used extensively in [25] to discuss the generating functional of the entire hierarchy of conserved quantities which are known to be a dual facet to the symmetry content of a system. Finally, in the present paper, a third interpretation is presented whereby these boundary conditions appear as canonical transformations of a certain type with respect to the new Poisson bracket we introduced. This was possible because we put both space and time coordinates on the same footing and considered a Poisson bracket corresponding to space evolution. By changing the roles of space and time, the defect boundary conditions now correspond to a canonical transformation of the fields. From this point of view, the conserved quantity (and therefore the symmetry) content "before" and "after" the transformation (i.e. on one side of the defect location and then on the other side) is the same. This can be seen for instance from eq (3.7) and its consequence (3.8) which show that the old and new conserved quantities only differ by constants which vanish under appropriate (time) boundary conditions. The symmetry content is not affected by the present Bäcklund type canonical transformations. In fact, this could be taken as an explanation of the integrable nature of such defect conditions.

This new theory will apply to other integrable classical field models (for instance the sine-Gordon model) or even discrete integrable models (like the Toda chain). We hope to return to these in the near future. Our result also opens, in principle, the way to the quantization of such models with defects. Indeed, when reformulated with the new Poisson structure, it is clear that the canonical property of the defect conditions is related to their origin in the form of Bäcklund transformations. On the other hand, the $\mathrm{r}$ matrix appearing in the time brackets is the same as that appearing in the usual space brackets. Therefore, it will be an interesting problem to understand how the quantization of these brackets and the quantization of Bäcklund transformations continue to intertwine so as to produce a 
quantum integrable system with integrable defect conditions. It is all the more interesting as quantum Bäcklund transformations are usually understood in connection with Baxter's $Q$ operator $[43,44]$.

Open Access. This article is distributed under the terms of the Creative Commons Attribution License (CC-BY 4.0), which permits any use, distribution and reproduction in any medium, provided the original author(s) and source are credited.

\section{References}

[1] B. McCoy and J. Perk, Two-spin correlation functions of an Ising model with continuous exponents, Phys. Rev. Lett. 44 (1980) 840.

[2] N.R. Jungwirth et al., A single-molecule approach to ZnO defect studies: single photons and single defects, arXiv:1402.1773.

[3] F. Nabarro, Theory of crystaline dislocation, Clarendon Press, Oxford U.K. (1967).

[4] S. Chandrasekhar and G. Ranganath, The structure and energetics of defects in liquid crystals, Adv. Phys. 35 (1986) 507.

[5] T. Lubensky et al., Topological defects and interactions in Nematic emulsions, cond-mat/9707133.

[6] D. Vollhardt and C. Wolfe, The phases of Helium 3, Taylor \& Francis (1990).

[7] G. Delfino, G. Mussardo and P. Simonetti, Statistical models with a line of defect, Phys. Lett. B 328 (1994) 123 [hep-th/9403049] [INSPIRE].

[8] G. Delfino, G. Mussardo and P. Simonetti, Scattering theory and correlation functions in statistical models with a line of defect, Nucl. Phys. B 432 (1994) 518 [hep-th/9409076] [INSPIRE].

[9] R. Konik and A. LeClair, Purely transmitting defect field theories, Nucl. Phys. B 538 (1999) 587 [hep-th/9703085] [INSPIRE].

[10] O.A. Castro-Alvaredo, A. Fring and F. Gohmann, On the absence of simultaneous reflection and transmission in integrable impurity systems, hep-th/0201142 [INSPIRE].

[11] M. Mintchev, E. Ragoucy and P. Sorba,, Reflection-transmission algebras, J. Phys. A 36 (2003) 10407.

[12] V. Caudrelier, M. Mintchev and E. Ragoucy, The quantum non-linear Schrödinger model with point-like defect, J. Phys. A 37 (2004) L367 [hep-th/0404144] [INSPIRE].

[13] V. Caudrelier, M. Mintchev and E. Ragoucy, Solving the quantum non-linear Schrodinger equation with delta-type impurity, J. Math. Phys. 46 (2005) 042703 [math-ph/0404047].

[14] Z. Bajnok and A. George, From defects to boundaries, Int. J. Mod. Phys. A 21 (2006) 1063 [hep-th/0404199] [INSPIRE].

[15] Z. Bajnok and O. el Deeb, Form factors in the presence of integrable defects, Nucl. Phys. B 832 (2010) 500 [arXiv:0909.3200] [INSPIRE].

[16] R. Weston, An algebraic setting for defects in the XXZ and sine-Gordon models, arXiv: 1006.1555 [INSPIRE]. 
[17] V. Caudrelier, M. Mintchev, E. Ragoucy and P. Sorba, Reflection-transmission quantum Yang-Baxter equations, J. Phys. A 38 (2005) 3431 [hep-th/0412159] [INSPIRE].

[18] P. Bowcock, E. Corrigan and C. Zambon, Classically integrable field theories with defects, Int. J. Mod. Phys. A 19S2 (2004) 82 [hep-th/0305022] [InSPIRE].

[19] P. Bowcock, E. Corrigan and C. Zambon, Affine Toda field theories with defects, JHEP 01 (2004) 056 [hep-th/0401020] [INSPIRE].

[20] E. Corrigan and C. Zambon, Aspects of sine-Gordon solitons, defects and gates, J. Phys. A 37 (2004) L471 [hep-th/0407199] [INSPIRE].

[21] E. Corrigan and C. Zambon, Jump-defects in the nonlinear Schrödinger model and other non-relativistic field theories, Nonlinearity 19 (2006) 1447 [nlin/0512038].

[22] E. Corrigan and C. Zambon, On purely transmitting defects in affine Toda field theory, JHEP 07 (2007) 001 [arXiv:0705.1066] [INSPIRE].

[23] E. Corrigan and C. Zambon, Comments on defects in the a(r) Toda field theories, J. Phys. A 42 (2009) 304008 [arXiv:0902.1307] [inSPIRE].

[24] E. Corrigan and C. Zambon, A new class of integrable defects, J. Phys. A 42 (2009) 475203 [arXiv:0908.3126] [INSPIRE].

[25] V. Caudrelier, On a systematic approach to defects in classical integrable field theories, IJGMMP 5 (2008) 1085 [arXiv:0704.2326].

[26] M.J. Ablowitz, D.J. Kaup, A.C. Newell and H. Segur, The inverse scattering transform fourier analysis for nonlinear problems, Stud. Appl. Math. 53 (1974) 249 [inSPIRE].

[27] I. Habibullin and A. Kundu, Quantum and classical integrable sine-Gordon model with defect, Nucl. Phys. B 795 (2008) 549 [arXiv:0709.4611] [INSPIRE].

[28] J. Avan and A. Doikou, Liouville integrable defects: the non-linear Schrödinger paradigm, JHEP 01 (2012) 040 [arXiv:1110.4728] [INSPIRE].

[29] J. Avan and A. Doikou, The sine-Gordon model with integrable defects revisited, JHEP 11 (2012) 008 [arXiv:1205.1661] [inSPIRE].

[30] A. Doikou, A note on $\mathrm{GL}_{N}$ type-I integrable defects, J. Stat. Mech. (2014) P02002.

[31] A. Doikou, Classical impurities associated to high rank algebras, Nucl. Phys. B 884 (2014) 142 [arXiv:1312.4786] [InSPIRE].

[32] T.J. Bridges, P.E. Hydon and J.K. Lawson, Multisymplectic structures and the variational bicomplex, Math. Proc. Camb. Phil. Soc. 148 (2010) 159.

[33] T. De Donder, Théorie invariante du calcul des variations, Gauthier-Villars, Paris France (1935).

[34] H. Weyl, Geodesic fields in the calculus of variations, Ann. Math. 36 (1935) 607.

[35] A. Kundu, Unraveling hidden hierarchies and dual structures in an integrable field model, arXiv:1201.0627 [INSPIRE].

[36] F. Magri, A simple model of the integrable Hamiltonian equation, J. Math. Phys. 19 (1978) 1156 [inSPIRE].

[37] E.K. Sklyanin, On complete integrability of the Landau-Lifshitz equation, LOMI E-79-3 (1980). 
[38] E.K. Sklyanin, The quantum version of the inverse scattering method, Zap. Nauchn. Sem. LOMI 95 (1980) 55.

[39] L.D. Faddeev and L.A. Takhtajan, Hamiltonian methods in the theory of solitons, Springer, Germany (2007).

[40] Y. Kodama and M. Wadati, Theory of canonical transformations for nonlinear evolution equations. 1, Prog. Theor. Phys. 56 (1976) 1740 [InSPIRE].

[41] Y. Kodama, Theory of canonical transformations for nonlinear evolution equations. II, Prog. Theor. Phys. 57 (1977) 1900.

[42] C. Zambon, The classical nonlinear Schrödinger model with a new integrable boundary, JHEP 08 (2014) 036 [arXiv: 1405.0967] [INSPIRE].

[43] M. Gaudin and V. Pasquier, The periodic Toda chain and a matrix generalization of the bessel function's recursion relations, J. Phys. A 25 (1992) 5243.

[44] E. Sklyanin, Bäcklund transformations and Baxters Q-operator, nlin/0009009. 